\title{
Correction to "Novel Small Molecule JP-153 Targets the Src-FAK-Paxillin Signaling Complex to Inhibit VEGF-Induced Retinal Angiogenesis"
}

In the above article [Toutounchian JJ, Pagadala J, Miller DD, Baudry, J, Park F, Chaum, E and Yates CR (2017) Mol Pharmacol 91:1-13], an author name was inadvertently omitted. The correct authors of the article are Jordan J. Toutounchian, Jayaprakash Pagadala, Duane D. Miller, Jerome Baudry, Frank Park, Edward Chaum, Vanessa Morales-Tirado, and Charles R. Yates.

In addition, the contributions of the added author, Vanessa Morales-Tirado, are as follows: contributed new reagents or analytic tools; performed data analysis; and wrote or contributed to the writing of the manuscript.

Finally, the current affiliation of Vanessa Morales-Tirado is as follows: Department of Ophthalmology, Department of Microbiology, Immunology \& Biochemistry, The University of Tennessee Health Science Center.

The authors regret this error and apologize for any confusion or inconvenience it may have caused. 